PROCEEDINGS OF THE

AMERICAN MATHEMATICAL SOCIETY

Volume 140, Number 10, October 2012, Pages 3531-3539

S 0002-9939(2012)11223-7

Article electronically published on February 23, 2012

\title{
POLYNOMIALS WITH ZEROS AND SMALL NORM ON CURVES
}

\author{
VILMOS TOTIK \\ (Communicated by Michael T. Lacey)
}

\begin{abstract}
This paper considers the problem of how zeros lying on the boundary of a domain influence the norm of polynomials (under the normalization that their value is fixed at a point). It is shown that $k$ zeros raise the norm by a factor $(1+c k / n)$ (where $n$ is the degree of the polynomial), while $k$ excessive zeros on an arc compared to $n$ times the equilibrium measure raise the norm by a factor $\exp \left(c k^{2} / n\right)$. These bounds are sharp, and they generalize earlier results for the unit circle which are connected to some constructions in number theory. Some related theorems of Andrievskii and Blatt will also be strengthened.
\end{abstract}

\section{RESUlts}

Let $C_{1}=\{z|| z \mid=1\}$ be the unit circle. The paper [12 discussed monic polynomials with prescribed zeros on $C_{1}$ having as small a norm as possible. The problem goes back to Turán's power sum method in number theory, in connection with which G. Halász [6] showed that there is a polynomial $Q_{n}(z)=z^{n}+\cdots$ with a zero at 1 and of norm $\left\|Q_{n}\right\|_{C_{1}} \leq \exp (2 / n)$, where $\|\cdot\|_{K}$ denotes the supremum norm on the compact set $K$. See [7] for the smallest possible norm for such a polynomial. Halász' result implies that if $Z_{1}, Z_{2}, \ldots, Z_{k_{n}}$ are arbitrary $k_{n}<n / 2$ points on the unit circle, then there is a $P_{n}=z^{n}+\cdots$ which has a zero at each $Z_{j}$ and has norm

$$
\left\|P_{n}\right\|_{C_{1}} \leq \exp \left(4 k_{n}^{2} / n\right) .
$$

It was shown in 12, Theorem 1] that, in general, one cannot have a smaller norm; namely, there is a constant $c>0$ with the following property: for any monic polynomials $P_{n}(z)=z^{n}+\cdots$,

(i) if $P_{n}$ has $k$ zeros (counting multiplicity) on $C_{1}$, then $\left\|P_{n}\right\|_{C_{1}} \geq 1+c(k / n)$,

(ii) if $P_{n}$ has $n|J| / 2 \pi+k$ zeros (counting multiplicity) on a subarc $J=J_{n}$ of the unit circle, then $\left\|P_{n}\right\|_{C_{1}} \geq \exp \left(c k^{2} / n\right)$.

The polynomial $Q_{[n / k]}\left(z^{k}\right)$ shows that (i) is sharp, and $Q_{[n / k]}(z)^{k}$ (and, say, $J$ a tiny interval around the point 1 ) shows that (ii) is sharp modulo constants.

For an alternative proof of part (ii), see the paper 4 by T. Erdélyi, where an improved version of a classical estimate of Erdős and Turán is used.

Another result of [12 showed that if the zeros are sufficiently well separated, then Halász' estimate can be improved. More precisely the following holds. Let

Received by the editors April 12, 2011.

2010 Mathematics Subject Classification. Primary 41A10, 31A15.

Key words and phrases. Polynomials, zeros, small supremum norm.

The author was supported by ERC grant No. 267055.

(C)2012 American Mathematical Society 
$\alpha>1$, and for each $n$ let there be given a set $X_{n}$ of $k_{n}$ points on the unit circle such that the distance between different points of $X_{n}$ is at least $\alpha 2 \pi / n$. Then there are polynomials $P_{n}(z)=z^{n}+\cdots$ such that $P_{n}$ vanishes at each point of $X_{n}$ and

$$
\left\|P_{n}\right\|_{C_{1}} \leq 1+D_{\alpha} \sqrt{k_{n} / n},
$$

where the constant $D_{\alpha}$ depends only on $\alpha$. In particular, if $k_{n}=o(n)$, then $\left\|P_{n}\right\|_{C_{1}}=1+o(1)$. We also mention that the conclusion is not valid for any $\alpha<1$; this follows from (ii) above. The estimate (2) was improved by Andrievskii and Blatt [3] to

$$
\left\|P_{n}\right\|_{C_{1}} \leq 1+D_{\alpha} k_{n} / n,
$$

which is a remarkable counterpart to (i) above.

By considering $z^{n} P_{n}(1 / z)$, all these have a formulation for polynomials $P_{n}$ with normalization $P_{n}(0)=1$, and this is the form to which the problem was generalized in [3] to analytic Jordan curves $\Gamma$ (multiple zeros) and in 2] to quasicircles (single zero). Note that if $\Gamma$ is a Jordan curve and $z_{0}$ is a fixed point inside $\Gamma$, then, by the maximum modulus theorem, we must have $\|P\|_{\Gamma} \geq 1$ for all polynomials $P$ with $P\left(z_{0}\right)=1$. We are interested in the problem of how zeros lying on $\Gamma$ influence this trivial lower estimate. For a single zero the analogue of Halász' result was settled even for quasicircles in the paper [2, where the zero can also occur, say, at a corner. For multiple zeros, Andrievskii and Blatt 3 proved that if $z_{0}$ is a fixed point inside the analytic curve $\Gamma$ and $P_{n}$ is a polynomial of degree $n$ with $k_{n}$ separated zeros on $\Gamma$, then

$$
\left\|P_{n}\right\|_{\Gamma} \geq 1+c k_{n} / n .
$$

On the other hand, if there are points $w_{1}, \ldots, w_{k_{n}}$ on $\Gamma$ which are well separated (in terms of a conformal mapping of the outer domain onto the exterior of the unit disk), then there is a $P_{n}$ of degree $n$ such that $P_{n}\left(z_{0}\right)=1$ and (3) holds (with $\|\cdot\|_{\Gamma}$ replacing $\left.\|\cdot\|_{C_{1}}\right)$.

The present paper was motivated by the aforementioned results of Andrievskii and Blatt, and, in particular, we will drop the analyticity assumption on $\Gamma$, as well as the separation assumption in (4). Actually, we shall prove the complete analogue of the results mentioned above for the unit circle for all $C^{1+\alpha}, \alpha>0$ Jordan curves. We emphasize that although the results match those for the unit circle, the proofs need ideas that do not use the special symmetry of the circle; in particular we cannot use trigonometric polynomials here. In fact, a Jordan curve can be pretty complicated from the point of view of polynomials. For example, it follows from the result below that the arc $I$ on $\Gamma$ depicted in Figure 1 tolerates many more zeros without raising the norm of a polynomial than the arc $J$ (more precisely, even though $I$ and $J$ have equal lengths, there is a $c$ such that $I$ can contain $c n$ zeros of a $P_{n}$ with $P_{n}\left(z_{0}\right)=1,\left\|P_{n}\right\|_{\Gamma}=1+o(1)$, while if a $P_{n}$ has the same number of zeros of $J$, then necessarily $\left\|P_{n}\right\|_{\Gamma} \geq \exp (d n)$, with some $d>0$, which is a very dramatic change).

Recall that $\Gamma$ is a Jordan curve if it is the homeomorphic image of the unit circle. It is called of class $C^{1+\alpha}$ if in its arc-length parametrization, the parameter function is differentiable and its derivative lies in the Lip $\alpha$ class.

Theorem 1. Let $\Gamma$ be a $C^{1+\alpha}$ Jordan curve, and let $z_{0}$ be a fixed point in the interior of $\Gamma$. If a polynomial $P_{n}$ of degree at most $n$ takes the value 1 at $z_{0}$ and 


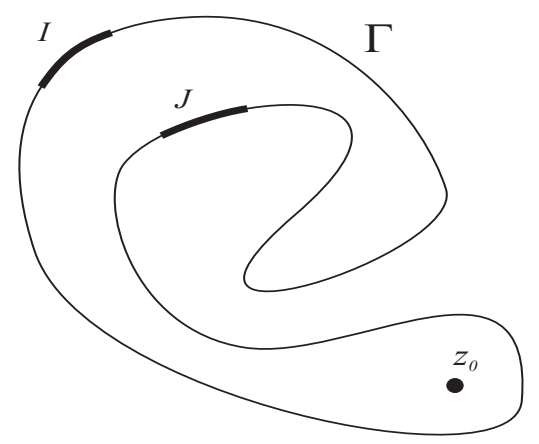

Figure 1. The arc $I$ has greater equilibrium measure than $J$, so there can be more zeros there without essentially raising the norm.

has $k_{n}$ zeros on $\Gamma$, then $\left\|P_{n}\right\|_{\Gamma} \geq 1+c k_{n} / n$ with a $c>0$ that depends only on $\Gamma$ and $z_{0}$.

This is sharp (at least for analytic $\Gamma$ ) because of the aforementioned result of Andrievskii and Blatt.

To formulate our next theorem, let $\mu_{\Gamma}$ be the equilibrium measure of $\Gamma$ (see e.g. [5], 10]). It is the unique unit Borel-measure on $\Gamma$ for which the logarithmic potential

$$
U^{\mu_{\Gamma}}(z)=\int \log \frac{1}{|z-t|} d \mu(t)
$$

is constant on $\Gamma$. One should think of $\mu_{\Gamma}$ as the distribution of a unit charge placed on the conductor $\Gamma$ when it is in equilibrium. Of course, if $\Gamma$ is the unit circle, then $\mu_{\Gamma}$ is just the normalized arc measure. Therefore, the following result is an extension of (ii) to smooth Jordan curves.

Theorem 2. Let $\Gamma$ be a $C^{1+\alpha}$ Jordan curve and $z_{0}$ be a fixed point inside $\Gamma$. If $P_{n}$ is a polynomial of degree at most $n$ such that $P_{n}\left(z_{0}\right)=1$ and $P_{n}$ has at least $k_{n}+n \mu_{\Gamma}(J)$ zeros on a subarc $J$ of $\Gamma$, then $\left\|P_{n}\right\|_{\Gamma} \geq \exp \left(c k_{n}^{2} / n\right)$ with a $c>0$ that depends only on $\Gamma$ and $z_{0}$.

Corollary 3. If $P_{n}$ are polynomials with $P_{n}\left(z_{0}\right)=1,\left\|P_{n}\right\|_{\Gamma}=1+o(1)$, then:

(a) $P_{n}$ has o(n) zeros on $\Gamma$.

(b) $P_{n}$ has at most $n \mu_{\Gamma}(J)+o(\sqrt{n})$ zeros on any subarc $J=J_{n}$ of the unit circle. In particular, if $w \in \Gamma$ is a zero of $P_{n}$, then its multiplicity is $o(\sqrt{n})$.

Next we show that Theorem 2 is sharp for all $C^{2}$ curves.

Theorem 4. Let $\Gamma$ be a $C^{2}$ Jordan curve and $z_{0}$ a point inside $\Gamma$. Then there are a constant $C$ and for every $w \in \Gamma$ and for every $n=1,2, \ldots$ a polynomial $P_{n, w}$ of degree at most $n$ such that $P_{n, w}\left(z_{0}\right)=1, P_{n, w}$ has a zero at $w$ and $\left\|P_{n, w}\right\|_{\Gamma} \leq$ $1+C / n$.

Corollary 5. Let $\Gamma$ be a $C^{2}$ Jordan curve and $z_{0}$ a point inside $\Gamma$. Then there is a constant $C$ with the following property: if $w_{1}, \ldots, w_{k_{n}} \in \Gamma$ are arbitrary $k_{n} \leq n$ points on $\Gamma$, then there is a polynomial $P_{n}$ of degree $n$ such that $P_{n}\left(z_{0}\right)=1, P_{n}$ has a zero at every $w_{j}$ and $\left\|P_{n}\right\|_{\Gamma} \leq \exp \left(C k_{n}^{2} / n\right)$. 
In particular, if $\left\{\delta_{n}\right\}$ is any positive sequence tending to 0 and if $w \in \Gamma$ is given, then there are polynomials $P_{n}$ with $P_{n}\left(z_{0}\right)=1,\left\|P_{n}\right\|_{\Gamma}=1+o(1)$ such that $w$ is a zero of $P_{n}$ of multiplicity $\geq \delta_{n} \sqrt{n}$. This shows that nothing more can be said about the multiplicities of zeros than what was stated in Corollary 3 .

It should be mentioned that the results are true for Dini smooth curves instead of $C^{1+\alpha}$-curves (for Dini smoothness, see 9]; it lies in between $C^{1}$ and $C^{1+\alpha}, \alpha>0$, smoothness). Indeed, using [9, Theorem 3.5] one can derive Proposition 6 below for Dini smooth curves, and the rest of the argument remains the same. One should also mention that even though the simple proof we give for Theorem 4 is valid only for $C^{2}$ curves, the result itself follows also from formula (3) in [2] actually for Dini smooth curves. The author is thankful for these remarks from the referee.

\section{Proof of Theorem 1}

We shall need the following facts from potential theory. For the necessary concepts (such as equilibrium measure, Green's function, etc.) from logarithmic potential theory, see e.g. [5] or [10]. Let $\Gamma$ be a Jordan curve and $\Omega$ the unbounded component of $\overline{\mathbb{C}} \backslash \Gamma$. As before, we denote by $\mu_{\Gamma}$ the equilibrium measure of $\Gamma$ and by $g_{\overline{\mathbb{C}} \backslash \Gamma}(z, \infty)$ the Green's function of $\Omega$ with pole at infinity. For a domain $G$ and a set $K \subset \partial G$, let $\omega(K, G, z)$ be the harmonic measure of $K$ in $G$ with respect to $z$ (i.e. $\omega(K, G, z)$ is the value at $z$ of the solution of the Dirichlet problem in $G$ with boundary function equal to 1 on $K$ and equal to 0 on the rest of the boundary). It is a unit Borel-measure on $\partial G$, and it is the unique measure on $\partial G$ for which the Poisson formula

$$
u(z)=\int u d \omega(\cdot, G, z)
$$

is valid for all $u$ that are harmonic in $G$ and continuous on $\bar{G}$. Harmonic measures are conformally invariant. For example, $\mu_{\Gamma} \equiv \omega(\cdot, \Omega, \infty)$ (see e.g. [10, Theorem 4.3.14]).

Proposition 6. Let $\Gamma$ be a $C^{1+\alpha}$ Jordan curve with some $0<\alpha<1$. The equilibrium measure $\mu_{\Gamma}$ has continuous (actually Lip $\alpha$ ) and positive density with respect to the arc measure on $\Gamma$. The same is true of all harmonic measures $\omega\left(\cdot, G, \zeta_{0}\right)$, $\zeta_{0} \in G$, where $G$ is either the bounded or the unbounded complement of $\Gamma$. Furthermore, the Green's function $g_{\overline{\mathbb{C}} \backslash \Gamma}(\cdot, \infty)$ of the unbounded component $\Omega$ with pole at infinity is uniformly Lip 1 (actually $C^{1+\alpha}$ ) on $\Gamma$.

Proof. These are well-known facts. For a reference to the statements concerning the equilibrium measure, see [14, Proposition 2.2]. Now using the fact that the equilibrium measure is the harmonic measure at infinity, i.e. $\mu_{\Gamma}(K)=\omega(K, \Omega, \infty)$ where $\Omega$ is the unbounded component of $\overline{\mathbb{C}} \backslash \Gamma$ (see e.g. [10, Theorem 4.3.14]), the claim concerning the harmonic measures also follows for $\omega(K, \Omega, \infty)$. But harmonic measures are conformally invariant, so the claim follows in general by using Möbius inversion: if $T \Gamma$ is the curve under the conformal map $T w=1 /(w-z)$, then $\omega(K, \Gamma, z)=\omega(T K, T \Omega, \infty)=\mu_{T \Gamma}(T K)$, and clearly this conformal map preserves $C^{1+\alpha}$-smoothness.

The statement concerning the Green's function follows from the KelloggWarschawski theorem (see [9, Theorems 3.5, 3.6]) stating that the conformal map $\varphi(z)=c z+d+e / z+\cdots, c>0$, from $\Omega$ onto the exterior of the unit disk is of class $C^{1+\alpha}$ in $\bar{\Omega}$, since $g_{\overline{\mathbb{C}} \backslash \Gamma}(z)=\log |\varphi(z)|$. 
Next, we need

Lemma 7. There are $\delta, \theta>0$ depending only on $\Gamma$ such that if $J=\widehat{a b}$ is a subarc of $\Gamma$ of length at most $\delta$ and if $P_{n}$ has at least $\theta n|J|$ zeros on $J$, then $\left|P_{n}(b)\right| \leq(1 / 3)\left\|P_{n}\right\|_{\Gamma}$.

Proof. According to the preceding proposition there is a $C_{1}$ such that for $t$ close to $\Gamma$,

$$
g_{\overline{\mathbb{C}} \backslash \Gamma}(t, \infty) \leq C_{1} \operatorname{dist}(t, \Gamma)
$$

and for any other $t$ this is automatically true. Hence, by the Bernstein-Walsh lemma [15, p. 77] for $\operatorname{dist}(t, \Gamma)<\rho$ we have

$$
\left|Q_{n}(t)\right| \leq e^{n g_{\bar{C} \backslash \Gamma}(t, \infty)}\left\|Q_{n}\right\|_{\Gamma} \leq e^{C_{1} n \rho}\left\|Q_{n}\right\|_{\Gamma}
$$

for any polynomial $Q_{n}$ of degree at most $n=1,2, \ldots$. Therefore, by Cauchy's formula,

$$
Q_{n}^{(m)}(z)=\frac{m !}{2 \pi i} \int_{|t-z|=\rho} \frac{Q_{n}(t)}{(t-z)^{m+1}} d t
$$

with integration on a circle with center at $z \in \Gamma$ and of radius $\rho$, we obtain for $z \in \Gamma$

$$
\left|Q_{n}^{(m)}(z)\right| \leq e^{C_{1} n \rho} m ! \frac{1}{\rho^{m}}\left\|Q_{n}\right\|_{\Gamma},
$$

and here $\rho>0$ is arbitrary.

Let $\delta$ be selected so that on any arc of $\Gamma$ of length at most $3 \delta$ the direction of the tangent line does not change more than $\pi / 8$. Let $J=\widehat{a b}$ be a subarc of $\Gamma$ of length at most $\delta$, and let $z_{1}, \ldots, z_{m}$ be the zeros of $P_{n}$ lying on $J$. Define the polynomial $Q_{n}$ as

$$
Q_{n}(z)=P_{n}(z) \prod_{j=1}^{m} \frac{z-a}{z-z_{j}} ;
$$

i.e. we move all the zeros of $P_{n}$ lying on $J$ into $a$ and leave all other zeros in place. Let $J^{\prime}=\widehat{a^{\prime} b^{\prime}}$ be the $\operatorname{arc}$ of $\Gamma$ that contains $J$ and for which $\left|\widehat{a^{\prime} a}\right|=\left|\widehat{b b^{\prime}}\right|=|J|$, where $|J|$ denotes the arc length of $J$ (i.e., $J^{\prime}$ is obtained by enlarging $J$ three times with respect to arc length). By considering the individual factors $|z-a| /\left|z-z_{j}\right|$, it is easy to see that $\left|P_{n}(b)\right| \leq\left|Q_{n}(b)\right|$, and if $z \notin \widehat{a^{\prime} b^{\prime}}, z \in \Gamma$, then $|z-a| /\left|z-z_{j}\right| \leq C_{2}$ with some $C_{2}$ depending only on $\Gamma$, and hence for such a $z$ we have $\left|Q_{n}(z)\right| \leq C_{2}^{m}\left|P_{m}(z)\right|$. Therefore, if the norm $\left\|Q_{n}\right\|_{\Gamma}$ is not attained on $J^{\prime}=\widehat{a^{\prime} b^{\prime}}$, which we are going to show under the assumption that there are sufficiently many zeros on $\widehat{a b}$, then

$$
\left\|Q_{n}\right\|_{\Gamma} \leq C_{2}^{m}\left\|P_{m}\right\|_{\Gamma} .
$$

Since $Q_{n}(z)$ has a zero at $a$ of order $m$, we have

$$
Q_{n}(z)=\int_{a}^{z} \int_{a}^{w_{1}} \cdots \int_{a}^{w_{m-1}} Q_{n}^{(m)}(w) d w d w_{m-1} \cdots d w_{1} .
$$

If $z=\gamma(s), s \in[0,|J|]$ is the arc length parametrization of $J$ with $\gamma(0)=a$, then this takes the form

$$
Q_{n}(z)=\int_{0}^{s} \int_{0}^{\tau_{1}} \cdots \int_{0}^{\tau_{m-1}} Q_{n}^{(m)}(\tau) \gamma^{\prime}(\tau) \gamma^{\prime}\left(\tau_{m-1}\right) \cdots \gamma^{\prime}\left(\tau_{1}\right) d \tau d \tau_{m-1} \cdots d \tau_{1} .
$$


Clearly, this formula also holds for $z \in J^{\prime}$ (with $s$ then in the extended range $[-|J|, 2|J|]$ ). Hence, $\left|\gamma^{\prime}(\tau)\right|=1$ and (5) with $\rho=\theta|J|$ gives for $z \in J^{\prime}$ (note that during $m$-fold integration the factor $1 / m$ ! emerges)

$$
\left|Q_{n}(z)\right| \leq e^{C_{1} n \theta|J|} m ! \frac{1}{(\theta|J|)^{m}} \frac{|\widehat{a z}|^{m}}{m !}\left\|Q_{n}\right\|_{\Gamma} \leq e^{C_{1} n \theta|J|}\left(\frac{2}{\theta}\right)^{m}\left\|Q_{n}\right\|_{\Gamma}
$$

since for $z \in J^{\prime}$ we have $|\widehat{a z}| \leq 2|J|$. Now if we assume that the number of zeros in $J$ is $m \geq \theta n|J|$, then we obtain for $z \in J^{\prime}$,

$$
\left|Q_{n}(z)\right| \leq e^{C_{1} m}\left(\frac{2}{\theta}\right)^{m}\left\|Q_{n}\right\|_{\Gamma}=\left(\frac{2 e^{C_{1}}}{\theta}\right)^{m}\left\|Q_{n}\right\|_{\Gamma}
$$

and for $\theta>2 e^{C_{1}}$ this means that the norm $\left\|Q_{n}\right\|_{\Gamma}$ is not attained in $J^{\prime}$, and so (6) is true. Therefore, we get from the preceding inequality and (6),

$$
\left|P_{n}(b)\right| \leq\left|Q_{n}(b)\right| \leq\left(\frac{2 e^{C_{1}}}{\theta}\right)^{m}\left\|Q_{n}\right\|_{\Gamma} \leq\left(\frac{2 e^{C_{1}} C_{2}}{\theta}\right)^{m}\left\|P_{n}\right\|_{\Gamma},
$$

from which the claim immediately follows if $\theta>6 e^{C_{1}} C_{2}$ (we may assume $m \geq 1$, for otherwise there is nothing to prove).

Proof of Theorem 1. In this proof $\omega\left(\cdot, z_{0}\right)$ denotes the harmonic measure in the interior of $\Gamma$. It follows from Proposition 6 that there is a constant $C_{0}$ such that for all $\operatorname{arcs} I$ on $\Gamma$ we have

$$
|I| \leq C_{0} \omega\left(I, z_{0}\right) .
$$

If $\left\|P_{n}\right\|_{\Gamma} \geq 3 / 2$, then we are ready. Otherwise consider the set $H$ of those $z \in \Gamma$ for which $\left|P_{n}(z)\right| \leq\left\|P_{n}\right\|_{\Gamma} / 2$. This set consists of a finite number of arcs, say $J_{1}, \ldots, J_{k}$, on which $\left|P_{n}(z)\right| \leq 3 / 4$. Since $\log \left|P_{n}(z)\right|$ is subharmonic, we have

$$
0=\log \left|P_{n}\left(z_{0}\right)\right| \leq \int \log \left|P_{n}\right| d \omega\left(\cdot, z_{0}\right)=\int_{H}+\int_{\Gamma \backslash H}=I_{1}+I_{2} .
$$

Now for any $j$,

$$
I_{1} \leq \omega\left(H, z_{0}\right) \log (3 / 4) \leq \omega\left(J_{j}, z_{0}\right) \log (3 / 4), \quad I_{2} \leq \log \left\|P_{n}\right\|_{\Gamma} ;
$$

hence the preceding inequalities give the theorem if one of the $J_{j}$ 's is of length greater than $\delta$ (with the $\delta$ from Lemma 7). Indeed, for then its harmonic measure is at least $\delta_{1}$ with some $\delta_{1}>0$ depending only on $\Gamma$ and $z_{0}$, and (8)-(9) give $\log \left\|P_{n}\right\|_{\Gamma} \geq c_{1}>0$.

If, on the other hand, all $J_{j}$ have length at most $\delta$, then, by Lemma 7 , the number of zeros of $P_{n}$ on $J_{j}$ is at most $\theta n\left|J_{j}\right|$, since the value of $P_{n}$ at the endpoints of $J_{j}$ is $\left\|P_{n}\right\|_{\Gamma} / 2$. Hence, using also (7), we have with some $C_{0}$,

$$
k_{n} \leq \theta n \sum_{j}\left|J_{j}\right| \leq \theta n C_{0} \sum_{j} \omega\left(J_{j}, z_{0}\right)=\theta n C_{0} \omega\left(H, z_{0}\right),
$$

and so from (8) and (9) we obtain

$$
\log \left\|P_{n}\right\| \geq I_{2} \geq-I_{1} \geq-\omega\left(H, z_{0}\right) \log (3 / 4) \geq\left(-\log (3 / 4) / \theta C_{0}\right) k_{n} / n,
$$

and this completes the proof. 


\section{Proof of Theorems 2 And 4}

Proof of Theorem 2. Assume, without loss of generality, that $z_{0}=0$. Apply the transformation $w=1 / z$, and let $\Gamma^{\prime}, J^{\prime}$ be the images of $\Gamma, J$ under this transformation; furthermore let $Q_{n}(z)=z^{n} P_{n}(1 / z)$. If $\omega^{\prime}(\cdot, 0)$ is the harmonic measure inside $\Gamma^{\prime}$ corresponding to the point 0 , then the logarithmic potential of $\omega^{\prime}(\cdot, 0)$ equals $\log 1 /|z|$ on and outside $\Gamma^{\prime}$ (consider e.g. that if $z$ is outside $\Gamma^{\prime}$, then $\log 1 /|z-t|$ is a harmonic function of $t$ inside $\Gamma^{\prime}$ ); hence on $\Gamma^{\prime}$,

$$
U^{n \omega^{\prime}(\cdot, 0)}(z)+\log \left|Q_{n}(z)\right| \leq \sup _{z \in \Gamma^{\prime}} \log \left|P_{n}(1 / z)\right|=\log \left\|P_{n}\right\|_{\Gamma} .
$$

Let $\nu_{n}$ be the normalized zero counting measure on the zeros of $Q_{n}$. Then $-\log \left|Q_{n}(z)\right|=U^{\nu_{n}}(z)$. Let $\tilde{\nu}_{n}$ be the balayage (see [11, Theorems II.4.1, II.4.4]) of $\nu_{n}$ out of the two components of $\overline{\mathbb{C}} \backslash \Gamma^{\prime}$ onto $\Gamma^{\prime}$; in other words, $\tilde{\nu}_{n}$ is the unique measure on $\Gamma$ that has total mass $n$ for which $U^{\tilde{\nu}_{n}}(z)=$ const $-\log \left|Q_{n}(z)\right|$ for all $z \in \Gamma$. Since taking the balayage out of a bounded region does not change the logarithmic potential on the boundary, while taking the balayage out of an unbounded region increases it by a positive constant on the boundary (see 11, Theorems II.4.1, II.4.4]), it follows that

$$
U^{\omega^{\prime}(\cdot, 0)}(z)-U^{\tilde{\nu}_{n}}(z) \leq \log \left\|P_{n}\right\|_{\Gamma}^{1 / n}, \quad z \in \Gamma^{\prime} .
$$

Since the left-hand side is harmonic outside $\Gamma^{\prime}$ (including $\infty$ ), this inequality holds outside $\Gamma^{\prime}$, as well. Therefore, we can apply the one-sided discrepancy theorem [1, Theorem 4.1.1] with $\beta=1$ to the curve $\Gamma^{\prime}$ and to the measure $\sigma=\omega^{\prime}(\cdot, 0)-\tilde{\nu}_{n}$ to conclude that for some $C>0$ and for any $\delta>0$,

$$
\left|\sigma\left(J^{\prime}\right)\right| \leq C\left(\delta^{-1 / 2}\left\|P_{n}\right\|_{\Gamma}^{1 / n}+\delta^{1 / 2}\right)
$$

from which, with

$$
\delta=\log \left\|P_{n}\right\|_{\Gamma}^{1 / n}
$$

we obtain

$$
\left|\sigma\left(J^{\prime}\right)\right| \leq 2 C \sqrt{\log \left\|P_{n}\right\|_{\Gamma}^{1 / n}} .
$$

Since the harmonic measure is conformal invariant, we have $\omega^{\prime}\left(J^{\prime}, 0\right)=\omega(J, \infty)=$ $\mu_{\Gamma}(J)$ (here the first harmonic measure is taken inside $\Gamma^{\prime}$, while the second one is taken in the unbounded component $\Omega$ of $\overline{\mathbb{C}} \backslash \Gamma$ ). Hence, by assumption, $Q_{n}$ has at least $k_{n}+\omega^{\prime}\left(J^{\prime}, 0\right)$ zeros on $J^{\prime}$, which implies that $\tilde{\nu}_{n}\left(J^{\prime}\right) \geq k_{n}+\omega^{\prime}\left(J^{\prime}, 0\right)$, and therefore $\left|\sigma\left(J^{\prime}\right)\right| \geq k_{n} / n$. Now the claim follows from (10).

Proof of Theorem 4. There is a polynomial $T_{N}=T_{N, w}$ of some degree $N$ such that the lemniscate set $L_{w}=\left\{z|| T_{N}(z) \mid=1\right\}$ consists of a single Jordan curve such that $L_{w}$ contains $\Gamma$ in its interior except for the point $w$, at which point $L_{w}$ and $\Gamma$ touch each other; see [8]. Furthermore, this is also true in the sense that a translatedrotated copy of $L_{w}$ can serve as $L_{w^{\prime}}$ for $w^{\prime} \in \gamma$ lying sufficiently close to $w$ (see [13, Theorem 2.3]). Then simple compactness tells us that there is a uniform bound on $N$, and the $T_{N, w}$ 's can be chosen in such a way that they are obtained by a linear transformation of the argument in a fixed finite family of polynomials. Let us refer 
to this fact by saying that the $T_{n, w}$ 's form a compact family. We may also assume that $T_{N, w}(w)=1$ (just multiply $T_{N, w}$ by a constant of modulus 1 if this is not the case).

Now let $Q_{m}$ be polynomials of degree $m=1,2, \ldots$ such that $Q_{m}(0)=1, Q_{m}(1)=$ 0 and $\left|Q_{m}(z)\right| \leq 1+4 / m$ (see [6]), and set

$$
P_{n, w}(z)=Q_{[n / N]}\left(T_{N, w}(z)\right) / Q_{[n / N]}\left(T_{N, w}\left(z_{0}\right)\right) .
$$

For this, $P_{n, w}(w)=0$. A simple calculation shows that by replacing a factor $z-a$ with $|a|<1$ in $Q_{m}(z)$ by $|a|^{2}(z-1 / \bar{a})$, we decrease the norm of $Q_{m}$ on the unit circle (keeping the normalization $Q_{m}(0)=1$ ), so we may assume that $Q_{m}$ has no zeros inside the unit circle. But then $\log \left|Q_{m}(z)\right|$ is harmonic in the unit disk; it takes the value 0 at the origin and has the bound $\leq \log (1+4 / m)$ throughout the disk. Hence we can derive from Harnack's inequality 10, Theorem 1.3.1] that for any compact subset $K$ of the open unit disk there is a constant $C_{K}$ such that $\left|Q_{m}(z)\right| \geq 1-C_{K} / m$ for $z \in K$. From the fact that the polynomials $T_{N, w}$ form a compact family it follows that the set $\left\{T_{N, w}\left(z_{0}\right) \mid w \in \Gamma\right\}$ lies in a fixed compact subset $K$ of the unit disk. Therefore, $Q_{[n / N]}\left(T_{N, w}\left(z_{0}\right)\right) \geq 1-2 C_{K} N / n$, and $\left|Q_{[n / N]}\left(T_{N, w}(z)\right)\right| \leq 1+8 N / n$ for $z \in \Gamma$, which show that $\left\|P_{n, w}\right\|_{\Gamma} \leq 1+C / n$ with some $C$.

\section{REFERENCES}

[1] V. V. Andrievskii and H-P. Blatt, Discrepancy of signed measures and polynomial approximation, Springer Monographs in Mathematics. Springer-Verlag, New York, 2002. MR.1871219 (2002k:30001)

[2] V. V. Andrievskii and S. Ruscheweyh, On polynomials with a prescribed zero on a quasicircle, Comput. Methods Funct. Theory, 8(2008), 243-259. MR2419476 (2009j:30009)

[3] V. V. Andrievskii and H-P. Blatt, Polynomials with prescribed zeros on an analytic curve, Acta Math. Hungar., 128(2010), 221-238. MR2671007(2011f:26023)

[4] T. Erdélyi, An improvement of the Erdős-Turán theorem on the distribution of zeros of polynomials, C. R. Math. Acad. Sci. Paris, 346(2008), 267-270. MR2414166 (2009e:30010)

[5] J. B. Garnett and D. E. Marshall, Harmonic measure, Cambridge University Press, New Mathematical Monographs, vol. 2, Cambridge, 2005. MR2150803(2006g:31002)

[6] G. Halász, On the first and second main theorem in Turán's theory of power sums, Studies in Pure Mathematics, 259-269, Birkhäuser, Basel, 1983. MR820228 (87d:11068)

[7] M. Lachance, E. B. Saff and R. Varga, Inequalities for polynomials with a prescribed zero. Math. Z., 168(1979), 105-116. MR544699 (80j:30009)

[8] B. Nagy and V. Totik, Sharpening of Hilbert's lemniscate theorem, J. D'Analyse Math., 96(2005), 191-223. MR2177185 (2006g:30008)

[9] Ch. Pommerenke, Boundary Behavior of Conformal Mappings, Grundlehren der mathematischen Wissenschaften, 299, Springer-Verlag, Berlin-Heidelberg-New York, 1992. MR 1217706 (95b:30008)

[10] T. Ransford, Potential Theory in the Complex Plane, Cambridge University Press, Cambridge, 1995. MR1334766 (96e:31001)

[11] E. B. Saff and V. Totik, Logarithmic Potentials with External Fields, Grundlehren der mathematischen Wissenschaften, 316, Springer-Verlag, New York-Berlin, 1997. MR 1485778 (99h:31001)

[12] V. Totik and P. Varjú, Polynomials with prescribed zeros and small norm, Acta Sci. Math., (Szeged) 73(2007), 593-612. MR2380067 (2009b:30012)

[13] V. Totik, Christoffel functions on curves and domains, Transactions of the Amer. Math. Soc., 362(2010), 2053-2087. MR2574887 (2011b:30006) 
[14] V. Totik, Asymptotics of Christoffel functions on arcs and curves (manuscript).

[15] J. L. Walsh, Interpolation and Approximation by Rational Functions in the Complex Domain, third edition, Amer. Math. Soc. Colloquium Publications, XX, Amer. Math. Soc., Providence, RI, 1960. MR0218587(36:1672a)

Bolyai Institute, Analysis Research Group of the Hungarian Academy of Sciences, University of Szeged, Szeged, Aradi v. tere 1, 6720, Hungary - And - Department of Mathematics and Statistics, University of South Florida, 4202 E. Fowler Avenue, PHY 114, TAMPa, FlORIDA 33620-5700

E-mail address: totik@mail.usf.edu 